\title{
Les perturbateurs thyroïdiens et leurs conséquences sur le développement cérébral
}

\author{
Jean-Baptiste Fini ${ }^{*}$ et Barbara Demeneix \\ Muséum National d'Histoire Naturelle, CNRS UMR 7221, Laboratoire Physiologie moléculaire de l'adaptation, 7 rue Cuvier, \\ 75005 Paris, France
}

Reçu le 31 janvier 2019

\begin{abstract}
Résumé-Une augmentation de la prévalence de nombreuses maladies affectant le système nerveux, aussi bien chez l'enfant que chez l'adulte a été rapportée. Certaines de ces maladies sont en relation avec des dysfonctionnements endocriniens et particulièrement de l'axe thyroïdien. Des troubles de l'attention ou l'hyperactivité, des syndromes liés à l'autisme, mais aussi la sclérose en plaques ou la maladie d'Alzheimer font partie de ces maladies qui peuvent survenir lors d'un dysfonctionnement du système thyroïdien. Il paraît de plus en plus évident qu'une exposition embryonnaire à des perturbateurs des hormones thyroïdiennes peut entraîner des conséquences à court et à long termes, non détectées par les diagnostics néonataux classiques. Les perturbateurs endocriniens comprennent une large gamme de molécules comme les plastifiants, certains, des surfactants ou retardants de flammes, dont plusieurs membres peuvent interférer avec la synthèse des hormones thyroïdiennes ou leurs actions. Nous reviendrons brièvement sur l'histoire de la perturbation endocrinienne, les exemples et les conséquences d'une exposition embryonnaire à de tels produits sur le développement du cerveau.
\end{abstract}

Mots clés :

hormones thyroïdiennes, perturbateurs endocriniens, neuro-développement, comportement, xénobiotiques

\begin{abstract}
Thyroid disruptors and their consequences on brain development and behavior. An increase in the prevalence of many diseases affecting the nervous system in both children and adults has been reported. Some of these diseases are related to endocrine dysfunction, notably of the thyroid axis. Examples in children are attention deficit/hyperactivity disorders and Autism Spectrum Disorders, diagnosed but most often affecting the whole life, and multiple sclerosis or Alzheimer's disease in adults. It is becoming increasingly clear that embryonic exposure to thyroid hormone disruptors can lead to short- and long-term consequences, that often escape conventional neonatal diagnosis. Endocrine disruptors comprise a wide range of molecules, plasticizers, some pesticides, surfactants, flame-retardants, etc., many of which can interfere with thyroid hormone synthesis or their actions. We here report briefly the history of endocrine disruptors, their properties and the consequences on neuronal development of embryonic exposure to some of them.
\end{abstract}

Keywords: thyroid hormones, endocrine disruptors, neuro-development, behavior, xenobiotics

Rachel Carson dans «Printemps silencieux» en 1962 écrivait «Comment des êtres intelligents pourraient-ils chercher à contrôler quelques espèces non désirées par une méthode qui contaminerait tout l'environnement et ferait craindre la maladie et la mort, même à leurs semblables ?». Elle faisait référence à l'insecticide DDT (dichlorodiphényltrichloroéthane) qui provoque l'amincissement des coquilles d'œufs. On peut y voir l'anticipation du concept «One World-One $H E A L T H \gg$ qui met en avant les liens entre santé

\footnotetext{
*Auteur correspondant: fini@mnhn.fr
}

humaine, santé animale et santé environnementale, mais nous pouvons également constater que presque 60 ans après, de nombreuses molécules «phytosanitaires » sont toujours mises sur le marché sans évaluation préalable de leurs effets sur le système endocrinien. Pourtant des effets «perturbateurs endocriniens » sur d'autres espèces que celles visées sont décrits dans la littérature scientifique. Nous nous intéresserons ici principalement aux impacts sur le développement cérébral et sur les molécules perturbant les hormones thyrö̈diennes, qui jouent un rôle déterminant pour un développement harmonieux du cerveau. 


\section{L'augmentation des maladies neuro- développementales}

L'augmentation des maladies neuro-développementales ou neuro-dégénératives sont rapportées dans de nombreuses études épidémiologiques (Rocca et al., 2011; Cerasuolo et al., 2017). Les dégénérescences cérébrales les plus fréquentes liées au vieillissement sont les maladies d'Alzheimer et de Parkinson, pour lesquelles le nombre de cas a augmenté depuis 30 ans (Mayeux \& Stern 2012). Chez les personnes âgées, la maladie d'Alzheimer est la principale forme de démence (60 à $70 \%$ des cas) (Goedert \& Spillantini, 2006). La forme familiale ou précoce de la maladie d'Alzheimer (5\% de cas) est corrélée à des mutations dans les gènes des protéines liées à la synthèse de l'amyloïde bêta : le précurseur de l'amyloïde (APP) et les présénilines (PSEN) (Piaceri et al., 2013). La seconde forme dite tardive ou sporadique est liée à certains facteurs de risques génétiques (polymorphismes de l'apolipoprotéine $\mathrm{E}$ ou de son récepteur neuronal, hyper-phosphorylation de la protéine Tau, etc.), mais aussi dans 25 à $40 \%$ des cas, à des facteurs extérieurs comme le vieillissement, un stress oxydant prolongé ou une altération de la fonction du protéasome au niveau cérébral (Halliwell \& Gutteridgo, 2007). Récemment, Canet et al. (2018) ont rapporté des corrélations entre des modulations des niveaux de glucocorticoïdes et de leurs récepteurs et l'augmentation de l'incidence de la maladie d'Alzheimer. Dès lors, des molécules chimiques perturbant cette voie de signalisation ou des voies de signalisations hormonales croisées telles que celles des hormones thyroïdiennes pourraient être reliées à certains cas de la maladie (Tan \& Vasan, 2009).

La maladie de Parkinson correspond à une mort des neurones dopaminergiques et peut apparaître avant l'âge de 40 ans ; ses causes restent floues. Des facteurs héréditaires interviendraient, mais l'utilisation ou le contact prolongé avec des pesticides agricoles organochlorés pourraient également jouer un rôle (Narayan et al., 2017).

La grande majorité des cas de maladies d'Alzheimer et de Parkinson sont observés chez des personnes âgées. L'exposition aux facteurs de risques peut avoir eu lieu des années, voire des décennies avant le diagnostic. De fait, l'identification d'un ou de plusieurs facteurs déclenchants est extrêmement complexe à réaliser sur la base d' études rétrospectives (Miller \& O'Callaghan 2008).

La sclérose en plaques (SEP), caractérisée par une perte progressive d'oligodendrocytes, est la maladie neurologique la plus fréquente chez les jeunes adultes. Une augmentation inexpliquée de l'incidence de la SEP a également été rapportée au cours des 20 dernières années (Grimaldi et al., 2007; Dilokthornsakul et al., 2016) et touche environ 750000 personnes en Europe (100 000 en France). Cette maladie neurodégénérative invalidante est caractérisée par une démyélinisation focale et disséminée des axones (Taveggia et al., 2010). La myélinisation est générée par les oligodendrocytes du système nerveux central tout au long de la vie des vertébrés et est un processus dépendant des hormones thyroïdiennes (HT) (Lee \& Petratos, 2016).
Les pathologies pour lesquelles les augmentations sont encore plus impressionnantes sont les troubles du spectre de l'autisme (TSA) et de l'attention avec hyperactivité. Les TSA regroupent divers syndromes, caractérisés par une communication verbale altérée et une absence d'interactions sociales, couplées à des gestes répétitifs. S'y ajoutent des critères secondaires tels que l'épilepsie et des déficits sensoriels ou une déficience intellectuelle. Aux États-Unis, en 1975, un enfant sur 5000 était diagnostiqué comme souffrant de TSA, tandis qu'en 2018, c'est un enfant sur 59 qui est enregistré comme tel (Baio et al., 2018). En France, le chiffre non officiel d'un jeune sur 100 est avancé. Weintraub (2011) a analysé les raisons possibles de cette augmentation. De nouvelles méthodes de diagnostic, une sensibilisation accrue et un âge croissant des parents sont des facteurs potentiels pris en compte qui pourraient expliquer environ $30 \%$ des nouveaux cas. Mais plus de $40 \%$ de ces derniers sont indépendants de ces facteurs et reste inexpliqués. Une origine génétique possible des TSA, avec une thérapie génique correctrice potentielle (Varga et al., 2017), a suscité un énorme intérêt au sein de la communauté des chercheurs dans les années 1990. Mais à ce jour, seuls 5 à $15 \%$ des cas de TSA ont été attribués à des mutations ponctuelles. Ici encore, l'origine environnementale est privilégiée pour expliquer l'augmentation de l'incidence de ces syndromes

\section{Les perturbateurs endocriniens dans la ligne de mire}

Parallèlement à l'augmentation de ces maladies neurodéveloppementales, dont les effets dépendent de l'âge, nous constatons une augmentation spectaculaire de la quantité et de la diversité des produits chimiques utilisés dans notre environnement (WHO/OMS, UNEP 2012). Bien entendu une corrélation n'est pas une cause et pour éprouver une hypothèse de cause à effet entre l'augmentation des produits chimiques et celle des maladies neurodéveloppementales, il faut des relevés d'exposition et des expériences utilisant des modèles pertinents dans un environnement contrôlé.

Les études montrant une imprégnation aux xénobiotiques sont disponibles et sans appel. Notre exposition aux produits chimiques a atteint un point où des dizaines de molécules exogènes différentes sont détectables dans le sang ou l'urine des personnes échantillonnées (Woodruff et al., 2011). Des méthodes de détection développées à partir d'autres matrices telles que les cheveux, les dents ou encore le liquide amniotique (Andra et al., 2015 ; JiménezDíaz et al., 2015; Appenzeller et al., 2017) ont permis de confirmer cette exposition universelle aux molécules exogènes (Braun, 2017). Parmi celles-ci, des centaines ont été identifiées comme «perturbateurs endocriniens » potentiels, capables d'imiter ou d'altérer les actions physiologiques des hormones. Les hormones dérivées du cholestérol (stéroïdes), d'amines (hormones thyrö̈diennes, HT), de lipides (prostaglandines) permettent une 
signalisation endocrine, c'est-à-dire la transmission d'un message chimique à distance de l'organe de synthèse. Ces hormones sont véhiculées par la circulation sanguine grâce à des protéines distributrices. Elles permettent de réguler leur propre production, généralement via un mécanisme de rétrocontrôle négatif au niveau de l'hypothalamus et de l'hypophyse. Au sein des différents tissus d'un organisme, elles régulent l'activation de la transcription de nombreux gènes au niveau cellulaire, via des récepteurs nucléaires capables de se lier à l'ADN sur des éléments de réponses spécifiques.

\section{L'origine et les caractéristiques des perturbateurs endocriniens}

En 1991, Theo Colborn avait rapporté des effets néfastes sur la faune, conséquents à la pollution aux polychloro-biphényls (PCB) des Grands Lacs. Ces produits, utilisés comme retardants de prise de feu sont extrêmement persistants. La pollution aigüe a entraîné une augmentation de la mortalité de la faune aquatique contaminée, mais la rémanence environnementale de quantités plus faibles de ces produits a mené à l'observation d'effets œstrogéno-mimétiques. On pensait, à cette époque, que le problème se résoudrait de lui-même avec la dilution progressive des niveaux de PCB. Mais la découverte, en 1991, d'un œstrogéno-mimétique pouvant migrer d'un polymère plastique (p-nonylphénol) par Ana Soto et Carlos Sonnenshein a poussé Théo Colborn à organiser la conférence de Wingspread afin de dresser un bilan général de l'état des connaissances. Théo Colborn conviait alors 21 scientifiques de divers horizons, toxicologues, endocrinologues, qui avaient mis en évidence divers effets de perturbations hormonales, résultant de l'exposition à des substances chimiques. Parmi cette vingtaine de chercheurs, Gray et al. (1985) montraient sur des modèles in vitro et in vivo les effets œestrogéniques du méthoxychlore (insecticide) ; Frédéric Vom Saal montrait l'impact sur le comportement à l'âge adulte, de faibles variations d'hormones stéroïdes transitant entre les sacs amniotiques de souriceaux frères et sœurs pendant la gestation (Vom Saal et al., 1991), tandis que le groupe de John Mc Lachlan montrait des effets à retardement sur le développement des organes génitaux à la suite de l'exposition au diéthylstilbestrol (Newbold et al., 1985). La preuve de principe que ces perturbations pouvaient également toucher l'Homme est venue des travaux du groupe d'Ana Soto et Carlos Sonnenschein, qui, après des recherches complémentaires, ont montré que le p-nonylphénol (agent de surface utilisé dans une nouvelle formulation de tubes en plastique employés au laboratoire) faisait proliférer des cellules humaines cancéreuses (MCF7) de manière similaire à l'hormone naturelle, le 17ß-œstradiol (Soto et al., 1991). L'observation chez l'Homme de ce potentiel hormono-mimétique a permis d'insérer dans un cadre global l'ensemble des relevés effectués sur la faune, mais aussi chez l'Homme, depuis une quarantaine d'années.
Depuis la formalisation de cette terminologie, les recherches sur les perturbateurs endocriniens se sont considérablement étendues tant sous l'aspect du volume que des thématiques. Les recherches ont pu affiner les paramètres caractérisant ces leurres hormonaux.

Tout d'abord l'idée d'une synergie de molécules a été suggérée par le groupe d'Andreas Kortenkamp; ces auteurs ont en effet relevé l'effet œstrogénique d'un mélange de huit molécules à des concentrations inférieures aux doses supposées sans effet (Silva et al., 2002). La synergie moléculaire n'a été démontrée que plus récemment, en 2015, par une équipe montpelliéraine (Delfosse et al., 2015) avec deux composés : un pesticide, le transnonachlore et une hormone de synthèse, l'éthinyl œstradiol. Par une cristallisation du récepteur avec les deux molécules, cette équipe a pu montrer que les deux molécules se stabilisent l'une l'autre au sein de la poche de liaison au ligand du récepteur nucléaire PXR.

Une deuxième caractéristique longtemps remise en cause par certains acteurs de l'industrie et toxicologues est l'existence des effets à faibles doses. Ce paramètre pourtant connu des endocrinologues et inhérent aux fonctions endocrine ou paracrine n'est pas en adéquation avec le dogme «tout est poison et rien n'est poison, c'est la dose qui fait le poison » énoncé par Paracelse. Cette idée, sous-entendant qu'un seuil au-dessous duquel aucun effet toxique n'existe, régit pourtant encore toute la législation actuelle sur les produits chimiques. Le principe de base de cette législation repose sur la recherche d'une dose sans effet observable chez l'animal (NOAEL) à partir de laquelle une dose journalière acceptable (DJA ou TDI Tolerable Daily Intake) est déterminée (pour revue, voir Hill et al., 2018).

Enfin, il existe des fenêtres de vulnérabilité aux perturbateurs endocriniens. En perturbant le développement in utero, où les hormones jouent des rôles cruciaux sur la migration, la prolifération et la différenciation cellulaires, les xénobiotiques peuvent engendrer des effets immédiats ou « retard » et donc visibles bien plus tard lors du développement. La période des 1000 premiers jours de vie, de la conception jusqu'à deux ans est une période cruciale qui va influencer la santé future de l'individu (Brumana et al., 2017). La théorie de l'origine développementale de la santé et des maladies (Developmental origin of Health and diseases ou DoHad) a été énoncée par Barker mais le concept est antérieur, comme la tragédie du diéthylstilbestrol l'illustre (Herbst et al., 1970). C'est également le concept déjà ancien des embryologistes de la plasticité phénotypique en fonction de l'environnement (Schneider, 1973).

Il est intéressant de constater que ce concept DoHad a été théorisé pour plusieurs voies hormonales. D'une part, Skakkebaek et al. (2001) a proposé la théorie selon laquelle une influence androgénique exercée par des polluants environnementaux pendant le développement foetal pourrait être à l'origine d'une dysgénésie testiculaire chez les enfants (triptyque cancer du testicule, cryptorchidie et hypospadias). Pour la voie œestrogénique, il a été 
montré que même des œestrogènes de faible «puissance», comme le bisphénol A (BPA) administré pendant l'organogenèse à des doses inférieures aux seuils réglementaires, entraînaient une différenciation avancée de la glande mammaire (Paulose et al., 2015). Enfin, étant donné le rôle crucial des hormones thyroïdiennes pour le développement harmonieux du cerveau et l'augmentation de nombreuses maladies neuro-développementales, ce concept DoHAd serait également applicable aux hormones thyroïdiennes et aux maladies neuro-développementales (Miller \& O'Callaghan 2008).

\section{Les dérèglements thyroïdiens}

Les premiers dérèglements thyroïdiens visibles par des goitres au niveau de la base du cou ont été décrits 3000 ans avant J.C. Il était alors recommandé de manger des crustacés ou des éponges bouillies. Nous savons que c'est l'iode contenu dans ces aliments qui permettait de pallier un apport en iode alors insuffisant. Un manque d'iode absolu pendant la grossesse chez la femme enceinte provoque une hypothyroïdie congénitale, non visible et qui, si elle n'est pas détectée et traitée à la naissance, peut provoquer le syndrome du crétinisme, causant de graves retards mentaux et des problèmes physiques (Legrand, 1982). L'exemple le plus connu est celui des «crétins des Alpes » : loin de toute source iodée, les femmes enceintes ne pouvaient pas synthétiser l'hormone thyroïdienne nécessaire au bon développement de leur foetus. Deux actions de Santé Publique menées dans les années 1970 ont permis d'éradiquer le crétinisme en Europe: d'une part, l'ajout d'iode dans le sel de table (Syed, 2015) et, d'autre part, le dépistage systématique de l'hypothyroïdie congénitale par dosage de la thyréostimuline (TSH) grâce au test du buvard, effectué trois jours après la naissance dans les maternités, mais dont les seuils sont toujours discutés (Lain et al., 2017).

Cette hypothyrö̈die congénitale peut être due à un problème développemental de la thyroïde (problème de migration des lobes) ou à un problème fonctionnel avec une glande dite «en place». Des travaux ont analysé les buvards en France sur une période de 20 ans et ont montré une augmentation significative du nombre d'hypothyroïdies congénitales avec glande en place, dans toutes les régions de France (Barry et al., 2016). Cette augmentation, sur un court intervalle de temps, suggère encore un rôle prépondérant de l'environnement, mais pose également la question des conséquences de variations prénatales faibles, indétectables par le test du buvard.

Alors que les enfants sont testés à la naissance pour une hypothyrö̈die congénitale, il n'existe à l'heure actuelle aucune mesure pour vérifier les niveaux hormonaux de la mère en pré-conceptuel ou durant la grossesse. Pourtant, de plus en plus d'études montrent l'impact fondamental de ces niveaux sur les capacités cognitives futures de l'enfant (Min et al., 2016). Ceci s'explique en partie par le fait que les hormones maternelles sont la seule source d'hormones thyroïdiennes pendant le premier trimestre de grossesse (pour revue, voir Korevaar et al., 2018). Par exemple,
Pop et al. (1999) ont montré que des niveaux faibles en $\mathrm{T}_{4}$ maternelle libre pendant le premier trimestre sont associés à un développement psychomoteur altéré chez l'enfant. Une hypothyroxinémie maternelle au début de la grossesse a également été associée, chez les enfants, à des temps de réaction allongés lors de tests cognitifs (Finken et al., 2013). Román et al. (2013) avaient montré l'association entre une hypothyroïdie maternelle et une incidence accrue de diagnostics de troubles autistiques chez les enfants à 6 ans. Plus récemment, une étude menée par le groupe de Robin Peeters sur la cohorte néerlandaise «Génération $\mathrm{R}$ » a montré un lien entre les niveaux de $\mathrm{T}_{4}$ libre chez la mère et le QI des enfants à l'âge de 6-8ans. Les corrélations montrent qu'entre 12 et $20 \mathrm{pmol} / \mathrm{L}$ de $\mathrm{T}_{4}$ libre chez la mère, les enfants ont le maximum de chance d'avoir un QI supérieur à 100 et le minimum de risque d'avoir un QI inférieur à 85. En cas d'hypo- comme d'hyper-thyroxinémie le risque d'abaissement du QI est fortement augmenté (Korevaar et al., 2016).

De nombreuses molécules chimiques sont détectées dans les compartiments foetaux, sang du cordon, liquide amniotique. La possibilité d'une perturbation thyroïdienne due à la présence d'une ou plusieurs de ces molécules durant le développement est donc une question fondamentale.

\section{Les niveaux d'action des perturbateurs thyroïdiens}

Les activités des hormones thyrö̈diennes (HT) sont multiples et leur rôle dans les mécanismes de régulation de la transcription lors du développement est activement étudié (pour revue, voir Flamant \& Samarut, 2003). Durant les trente dernières années, de nombreuses études ont permis d'affiner le rôle des HT. Chez l'Homme, les HT sont essentielles au développement des organes (cerveau, cœur, reins, muscle squelettique, oreille interne, œil) (Sohmer \& Freeman, 1996), mais également à un bon réglage du métabolisme énergétique (Kadenbach et al., 1995). Les HT sont impliquées dans le développement du système nerveux (Bernal, 2007) et en particulier dans la différenciation des neurones (López-Juárez et al., 2012). Des études récentes ont montré qu'une absence d'HT conduit à un engagement dans la voie oligodendrocytaire (Remaud et al., 2017). Contrairement à ce que l'on pensait sur la diffusion facilitée des HT au travers de la double couche phospholipidique, des transporteurs membranaires spécifiques des hormones thyroïdiennes (MCT8, OATP) jouent un rôle majeur dans l'internalisation ou l'externalisation cellulaire des HT (Dumitrescu et al., 2004 ; Friesema et al., 2004).

Il est donc raisonnable de formuler l'hypothèse selon laquelle des perturbations chimiques, lors de la mise en place de ces tissus, organes ou fonctions, peuvent avoir lieu à différents niveaux tels que :

- la régulation du rétrocontrôle des hormones au niveau central ;

- la synthèse des hormones thyroïdiennes au niveau de la glande;

- la distribution des hormones via la circulation sanguine; 
- le métabolisme périphérique des HT (désiodation, glucuronidation, sulfatation);

- le métabolisme cellulaire des hormones;

- la liaison des HT sur leurs récepteurs.

L'impact de ces perturbations peut entraîner une modification des fonctions sous le contrôle des hormones thyroïdiennes (transcription, prolifération, neurogenèse, gliogenèse, migration) qui peuvent avoir des répercussions sur la structure du cerveau et par conséquent des modifications comportementales (WHO/OMS, 2012). La multiplication active des cellules gliales, la myélinisation, la croissance massive d'arbres dendritiques neuronaux et la connectivité synaptique vont influencer massivement cette structure cérébrale (Lefebvre et al., 2015). Les conditions pendant la vie fotale et les deux premières années suivant la naissance vont également influencer la taille définitive du cerveau. Il a été montré, chez la souris adulte, que les HT favorisent l'engagement des cellules souches neurales (CSN) dans la voie neuronale (Fini et al., 2012b; López-Juárez et al., 2012), et que des mécanismes bloquant leur action sont nécessaires pour la génération des précurseurs des oligodendrocytes à partir des CSN (Remaud et al., 2017).

\section{Les conséquences d'une perturbation précoce sur le développement du cerveau}

La complexité des questions posées par la perturbation hormonale conjuguée à la multiplicité des effets des HT, qui peuvent différer entre espèces, rendent les résultats d'études sur modèles in vitro et in vivo difficiles à interpréter pour l'Homme. C'est pourquoi l'OCDE a insisté, par un communiqué datant d'octobre 2014, sur l'importance capitale de développer des approches spécifiques permettant la détection de xénobiotiques agissant sur l'axe thyroïdien. Il est important de noter que la poche de liaison au ligand du récepteur aux HT (TR) est extrêmement spécifique et que, contrairement au processus existant pour la plupart des autres hormones, la voie majoritaire d'action des perturbateurs thyroïdiens n'est pas le récepteur nucléaire (Dietrich et al., 1977).

Plusieurs revues ont montré que de nombreuses molécules étaient des perturbateurs thyroïdiens potentiels (Brucker-Davis, 1998; Howdeshell, 2002). En 2015, une étude a estimé à 157 milliards d'euros le coût annuel en Europe de l'absence de législation sur certains produits aux propriétés perturbatrices endocriniennes (Bellanger et al., 2015). Sur ces 157 milliards, 120 milliards sont attribués à l'utilisation de certains pesticides tels que le chlorpyrifos. En 2013, l'Agence de sécurité alimentaire européenne (EFSA) a rapporté que 101 des 287 pesticides examinés avaient potentiellement une action de perturbation thyroïdienne.

\section{Les pesticides}

Le chlorpyrifos est un insecticide organophosphoré utilisé pour lutter contre les moustiques, les mouches dans les champs de maïs et autrefois contre les insectes domestiques (US EPA, 2000a). Sa production est estimée à 100000 tonnes/an (INERIS, 2006). En France, le chlorpyrifos est également utilisé en mélange pour pulvérisation aérienne sur divers végétaux comme les arbres fruitiers. Une étude mesurant les métabolites urinaires de pesticides au cours du troisième trimestre chez 386 femmes enceintes a indiqué que l'exposition au chlorpyrifos était prévalente ( $42 \%$ des femmes avaient des niveaux détectables de métabolites de chlorpyrifos) (Berkowitz et al., 2003). Le principal effet toxique connu du chlorpyrifos est l'inhibition de l'acétylcholinestérase mais d'autres effets de perturbations endocriniennes ont été rapportés. Le chlorpyrifos provoque la perte de cellules du cerveau chez les jeunes rats (Campbell et al., 1997), et l'exposition prénatale au chlorpyrifos chez l'Homme a été corrélée à une réduction significative du QI chez les enfants et une modification de la structure cérébrale (Rauh et al., 2006, 2011, 2012). Récemment, l'équipe de V. Laudet a montré que la maturation du poisson-chirurgien (Acanthurus triostegus) est contrôlée par les HT et perturbée par le chlorpyrifos. En empêchant leur maturation, le chlorpyrifos réduit la capacité des juvéniles à brouter les algues qui altèrent les coraux. C'est tout un écosystème qui se trouve déséquilibré (Holzer et al., 2017). Notre groupe de recherches a également pu montrer, dans une étude à plus long terme, que tous les têtards de Xenopus laevis exposés pendant la mise en place de la neurogenèse pouvaient effectuer leur métamorphose, mais que les cerveaux des animaux exposés au chlorpyrifos montraient une morphologie différente. De façon notable et reproductible, la distribution des diamètres des neurones est modifiée, les animaux exposés pendant la période embryonnaire ayant des diamètres axonaux plus petits (Spirhanzlova et al., soumis).

Ce pesticide est particulièrement intéressant car son autorisation d'usage en Europe est en cours de réévaluation avec un avis rendu en 2019. Des éléments rapportés par Mie et al. en novembre 2018 montrent que les données fournies par Dow Chemicals lors de l'évaluation initiale du chlorpyrifos étaient volontairement trompeuses.

D'autres pesticides peuvent agir sur l'axe thyrö̈dien comme l'amitrole (amniotriazole), un herbicide non sélectif, l'imidaclopride, un pyréthroïde ou le DDT précédemment évoqué.

Le mécanisme d'action de l'amitrole est d'inhiber la synthèse des caroténoïdes, la formation de la chlorophylle, et de limiter la repousse de bourgeons. L'amitrole a été banni d'utilisation dans les cultures vivrières, dans les années 1970 aux États-Unis, après que des études ont montré son rôle dans la survenue de tumeurs de la thyroïde chez des rongeurs (Tsuda et al., 1976). Son effet antithyroïdien a été largement documenté (Brucker-Davis 1998). En 2003, Hosoda et al. ont montré une inhibition de la formation de synapses induite par les HT à la suite d'un traitement à l'amitrole. Son mode d'action le plus probable est une inhibition de la thyroïde peroxydase (TPO) (Li et al., 2009).

L'imidaclopride a été le premier pesticide néonicotinoïde mis sur le marché et, en 2009, l'insecticide le plus utilisé au monde. En 2013, sur la base de rapports 
scientifiques montrant un effet dramatique des néonicotinoïdes sur la survie des abeilles, la Commission européenne a décidé d'interdire la mise sur le marché de graines enrobées ainsi que l'utilisation sur les plantes attirant les insectes pollinisateurs (règlement UE 485/2013 de la Commission du 24 mai 2013 (JO L139/12)). Cependant, son utilisation après la floraison continue d'être approuvée sur les céréales. Une étude de Millot et al. (2017) a montré les effets de l'imidaclopride sur d'autres acteurs de l'écosystème, en particulier l'empoisonnement des oiseaux, élargissant les effets déjà répertoriés sur les abeilles. La fiche toxicologique de l'INRS sur l'imidaclopride mentionne que l'administration chronique d'imidaclopride est responsable d'effets anti-thyroïdiens chez le rat (Moser et al., 2015). Ces résultats sont étayés par des publications montrant des effets potentiels de l'imidaclopride sur la reproduction et sur l'homéostasie des hormones thyroïdiennes (Pandey \& Mohanty, 2015). De plus, une diminution de l'activité motrice/locomotrice a été notée chez le rat lors d'une étude de neurotoxicité par administration unique durant la gestation des rattes gravides (Abou-Donia et al., 2008).

Enfin, le DDT et son métabolite principal le 4-4'-DDE sont encore détectés chez de très nombreux individus, près de 40 ans après son interdiction. En 2013, Carmen Freire et al. ont montré que ces molécules étaient toujours détectables dans les organismes de populations fortement exposées, comme les Brésiliens, chez lesquels elles altéraient les fonctions thyroïdiennes et cognitives (Freire et al., 2013).

Plusieurs autres catégories de molécules interfèrent avec la signalisation par les HT. Les retardateurs de flamme présents dans tous les produits électroniques, batteries, meubles, tels que le tétrabromobisphénol A (TBBPA) et les polybromodiphényl-éthers (PBDE) contiennent un ou plusieurs atomes de brome qui peuvent interférer avec le métabolisme des hormones thyroïdiennes (Fini et al., 2012a; Hoffman et al., 2017). Des niveaux élevés de PBDE sont positivement corrélés aux niveaux de TSH, tandis que de faibles niveaux de PBDE sont associés avec des taux élevés de $\mathrm{T}_{3}$ ou $\mathrm{T}_{4}$ (Zheng et al., 2017). La diminution des taux de $\mathrm{T}_{4}$ est toutefois l'effet négatif le plus systématique dans les populations, en fonction de l'exposition aux PBDE. Une diminution des taux de TH circulants a également été observée dans des études expérimentales sur des rongeurs, des poissons et des oiseaux après exposition périnatale à du PBDE (Richardson et al., 2008; Lee et al., 2010 ; Costa et al., 2014).

\section{Quelques autres exemples}

D'autres molécules, telles que les polychlorobiphényles utilisés comme retardants de flamme jusque dans les années 1970, montrent également des effets de perturbation des hormones thyroïdiennes. Par exemple, Jacobson et Jacobson (1996) ont montré que les concentrations sanguines en PCB étaient inversement corrélées avec les niveaux circulants de $\mathrm{T}_{4}$. Les enfants des femmes les plus contaminées avaient des QI moins élevés à l'âge de 11 ans. Ces résultats ré-analysés récemment semblent être pertinents (Stewart et al., 2012).
Les phtalates sont des plastifiants, qui représentaient encore $70 \%$ du marché en 2010. Ils étaient utilisés dans les jouets jusqu'en 2005 en Europe et dans les tubulures employées en médecine jusqu'en 2017. Ils sont toujours présents dans les poches de sang stockées et nécessaires aux transfusions. Les phtalates affectent le métabolisme du foie et des lipides, et la majorité des études sur les perturbations hormonales ont révélé des effets antiandrogéniques et anti-thyroïdiens. Des études épidémiologiques ont aussi montré un lien entre les taux de certains métabolites des phtalates chez la mère et des retards de neuro-développement chez les enfants (Wu et al., 2017).

Enfin, les métaux lourds peuvent aussi perturber le fonctionnement hormonal. Un exemple édifiant est celui du méthylmercure, un neurotoxique à forte dose, qui s'accumule dans les tissus des prédateurs marins, que nous consommons. À faible dose, il diminue aussi l'activité de certaines enzymes fonctionnant avec du sélénium telles que les désiodases. Une perturbation des désiodases peut modifier l'apport local en HT dans les cellules neurales et modifier le devenir cellulaire et la mise en place des structures du cerveau (Soldin et al., 2008).

D'autres composés aux conséquences semblables sont les composés phénoliques. Parmi ces composés, on retrouve le bisphénol A et ses substituts (les bisphénols F et S), utilisés comme plastifiants, mais aussi le triclosan, un bactéricide utilisé dans le dentifrice et les produits ménagers ainsi que les benzophénones, les filtres antiultra-violets des crèmes solaires. Le triclosan et certaines benzophénones perturbent bien la signalisation thyroïdienne et auraient des conséquences sur les fonctions cognitives (Köhrle, 2000).

\section{Les mélanges}

Enfin, la question cruciale des effets de mélanges de toutes ces molécules se pose. Cette problématique n'est pas prise en considération par la législation actuelle.

Notre groupe de recherche a testé quinze molécules appartenant aux différentes catégories précédemment citées. Nous avons testé sur des têtards le potentiel de perturbation thyroïdienne de molécules uniques à différentes concentrations (Fini et al., 2017). Onze molécules sur quinze ont montré une activité perturbatrice des hormones thyroïdiennes aux concentrations testées. Il est à noter que tous les composés contenant un atome halogéné (fluor, brome, chlore) ont montré une activité de perturbation thyrö̈dienne. Parmi celles-ci : le triclosan, le diéthyl-hexyl-phthalate (DEHP), les pesticides organochlorés, le PFOA, le PCB 153, le BDE-209 ou encore le méthyl-mercure. Nous avons ensuite créé un mélange de ces quinze molécules aux concentrations mesurées dans le liquide amniotique (basées sur la littérature). Nous avons testé le mélange soit à la concentration amniotique, soit 10 fois moins ou 10 fois plus concentré. Nous avons montré que ce mélange perturbe la signalisation des hormones thyrö̈diennes. L'expression de nombreux gènes du cerveau a été réprimée par l'exposition à ce mélange, notamment les gènes tubb2b (marqueur de neurones 
matures) et $m b p$ (marqueur d'oligodendrocytes matures) à la plus forte concentration. Nous avons alors étudié la fonctionnalité et la structure des cerveaux de ces têtards.

Afin de visualiser le cerveau entier et de quantifier les populations neuronales et oligodendrocytaires, ainsi que leurs volumes cellulaires respectifs, nous avons rendu transparents les cerveaux des têtards grâce à une collaboration avec l'équipe TEFOR (Affaticati et al., 2018)

Nous avons ensuite travaillé en microscopie confocale, sur des têtards doublement transgéniques $\mathrm{Tg}$ ( $M m u-M b p$ : gfp,Nbt: DsRed), en utilisant des marqueurs fluorescents traçant en vert les corps cellulaires des oligodendrocytes matures et en rouge les neurones matures. L'effet du mélange précédemment cité a été étudié sur une partie du cerveau, où les deux populations cellulaires, oligodendrocytes et neurones sont présentes. L'exposition au mélange a induit une modification du volume des neurones et des oligodendrocytes. Enfin, nous avons pu montrer une baisse significative du comportement de nage des têtards (distance parcourue) après une exposition au mélange de ces quinze molécules (Fini et al., 2017).

\section{Conclusions}

Voici donc quelques exemples de perturbations thyroïdiennes, avec pour certains des conséquences au niveau neural (pour une revue exhaustive sur ces types de molécules, voir Mughal et al., 2018). Parce que de simples dosages ne suffisent pas pour prévoir des conséquences «retard» à cause des faibles doses ou parce qu'elles dépendent du moment d'exposition, il est réellement nécessaire d'utiliser des tests adéquats, non seulement pour identifier les molécules ayant une activité de perturbation endocrinienne, mais aussi pour évaluer les molécules seules ou en «mélange». Le cerveau humain représente une des structures les plus complexes et les plus fascinantes que l'évolution ait façonné ; à nous aujourd'hui de le protéger et de définir les principaux acteurs impliqués dans l'augmentation inédite de maladies neuro-développementales.

\section{Références}

Abou-Donia, M.B., Goldstein, L.B., Bullman, S., Tu, T., Khan, W.A., Dechkovskaia, A.M., Abdel-Rahman, A.A. (2008). Imidacloprid induces neurobehavioral deficits and increases expression of glial fibrillary acidic protein in the motor cortex and hippocampus in offspring rats following in utero exposure. J Toxicol Environ Health A, 71, 119-130.

Affaticati, P., Le Mével, S., Jenett, A., Rivière, L., Machado, E., Mughal, B.B., Fini, J.B. (2018). X-FaCT: Xenopus-Fast Clearing Technique. Methods Mol Biol, 1865, 233-241.

Andra, S.S., Austin, C., Arora, M. (2015). Tooth matrix analysis for biomonitoring of organic chemical exposure: Current status, challenges, and opportunities. Environ Res, 142, 387-406.
Appenzeller, B.M.R., Hardy, E.M., Grova, N., Chata, C., Faÿs, F., Briand, O., Schroeder, H., Duca, R.C. (2017). Hair analysis for the biomonitoring of pesticide exposure: comparison with blood and urine in a rat model. Arch Toxicol, 91, 2813-2825.

Baio, J., Wiggins, L., Christensen, D.L., Maenner, M.J., Daniels, J., Warren, Z., Kurzius-Spencer, M., Zahorodny, W., Robinson Rosenberg, C., White, T., Durkin, M.S., Imm, P., Nikolaou, L., Yeargin-Allsopp, M., Lee, L.C., Harrington, R., Lopez, M., Fitzgerald, R.T., Hewitt, A., Pettygrove, S., Constantino, J.N., Vehorn, A., Shenouda, J., Hall-Lande, J., Van Naarden Braun, K., Dowling, N.F. (2018). Prevalence of autism spectrum disorder among children aged 8 yearsAutism and Developmental Disabilities Monitoring Network, 11 Sites, United States, 2014. MMWR Surveill Summ, 67, $1-23$.

Barry, Y., Bonaldi, C., Goulet, V., Coutant, R., Léger, J., Paty, A.C., Delmas, D., Cheillan, D., Roussey, M. (2016). Increased incidence of congenital hypothyroidism in France from 1982 to 2012: a nationwide multicenter analysis. Ann Epidemiol, 26, 100-105.e4.

Bellanger, M., Demeneix, B., Grandjean, P., Zoeller, R.T., Trasande, L. (2015). Neurobehavioral deficits, diseases, and associated costs of exposure to endocrine-disrupting chemicals in the European Union. J Clin Endocrinol Metab, 100, 1256-1266.

Berkowitz, G.S., Obel, J., Deych, E., Lapinski, R., Godbold, J., Liu, Z., Landrigan, P.J., Wolff, M.S. (2003). Exposure to indoor pesticides during pregnancy in a multiethnic, urban cohort. Environ Health Perspect, 111, 79-84.

Bernal, J. (2007). Thyroid hormone receptors in brain development and function. Nat Clin Pract Endocrinol Metab, 3, 249-259.

Braun, J.M. (2017). Early-life exposure to EDCs: role in childhood obesity and neurodevelopment. Nat Rev Endocrinol, 13, 161-173.

Brucker-Davis, F. (1998). Effects of environmental synthetic chemicals on thyroid function. Thyroid, 8, 827-856.

Brumana, L., Arroyo, A., Schwalbe, N.R., Lehtimaki, S., Hipgrave, D.B. (2017). Maternal and child health services and an integrated, life-cycle approach to the prevention of non-communicable diseases. BMJ Glob Health, 2(3), e000295.

Campbell, C.G., Seidler, F.J., Slotkin, T.A. (1997). Chlorpyrifos interferes with cell development in rat brain regions. Brain Res Bull, 43, 179-189.

Canet, G., Chevallier, N., Zussy, C., Desrumaux, C., Givalois, L. (2018). Central role of glucocorticoid receptors in Alzheimer's disease and depression. Front Neurosci, 12, 739.

Carson, R. (1962). Silent Spring, Houghton Mifflin. «Printemps silencieux », traduit de «Silent Spring », Plon, Paris.

Cerasuolo, J.O., Cipriano, L.E., Sposato, L.A., Kapral, M.K., Fang, J., Gill, S.S., Hackam, D.G., Hachinski, V. (2017). Population-based stroke and dementia incidence trends: Age and sex variations. Alzheimers Dement, 13, 1081-1088.

Colborn, T. (1991). Epidemiology of Great Lakes bald eagles. $J$ Toxicol Environ Health, 33, 395-453.

Costa, L.G., de Laat, R., Tagliaferri, S., Pellacani, C. (2014). A mechanistic view of polybrominated diphenyl ether (PBDE) developmental neurotoxicity. Toxicol Lett, 230, 282-294.

Delfosse, V., Dendele, B., Huet, T., Grimaldi, M., Boulahtouf, A., Gerbal-Chaloin, S., Beucher, B., Roecklin, D., Muller, C., Rahmani, R., Cavaillès, V., Daujat-Chavanieu, M., Vivat, V., Pascussi, J.M., Balaguer, P., Bourguet, W. (2015). Synergistic activation of human pregnane $\mathrm{X}$ receptor by binary cocktails of pharmaceutical and environmental compounds. Nat Commun, 6, 8089. 
Dietrich, S.W., Bolger, M.B., Kollman, P.A., Jorgensen, E.C. (1977). Thyroxine analogues. 23.Quantitative structureactivity correlation studies of in vivo and in vitro thyromimetic activities. J Med Chem, 20, 863-880.

Dilokthornsakul, P., Valuck, R.J., Nair, K.V., Corboy, J.R., Allen, R.R., Campbell, J.D. (2016). Multiple sclerosis prevalence in the United States commercially insured population. Neurology, 86, 1014-1021.

Dumitrescu, A.M., Liao, X.H., Best, T.B., Brockmann, K., Refetoff, S. (2004). A novel syndrome combining thyroid and neurological abnormalities is associated with mutations in a monocarboxylate transporter gene. Am J Hum Genet, 74, 168-175.

Fini, J.B., Riu, A., Debrauwer, L., Hillenweck, A., Le Mével, S. Chevolleau, S., Boulahtouf, A., Palmier, K., Balaguer, P., Cravedi, J.P., Demeneix, B.A., Zalko, D. (2012a). Parallel biotransformation of tetrabromobisphenol A in Xenopus laevis and mammals: Xenopus as a model for endocrine perturbation studies. Toxicol Sci, 125, 359-367.

Fini, J.B., Le Mevel, S., Palmier, K., Darras, V.M., Punzon, I., Richardson, S.J., Clerget Froidevaux, M.S., Demeneix, B.A. (2012b). Thyroid hormone signaling in the Xenopus laevis embryo is functional and susceptible to endocrine disruption. Endocrinology, 153, 5068-5081.

Fini, J.B., Mughal, B.B., Le Mével, S., Leemans, M., Lettmann, M., Spirhanzlova, P., Affaticati, P., Jenett, A., Demeneix, B. A. (2017). Human amniotic fluid contaminants alter thyroid hormone signalling and early brain development in Xenopus embryos. Sci Rep, 7, 43786.

Finken, M.J., van Eijsden, M., Loomans, E.M., Vrijkotte, T.G., Rotteveel, J. (2013). Maternal hypothyroxinemia in early pregnancy predicts reduced performance in reaction time tests in 5- to 6-year-old offspring. J Clin Endocrinol Metab, 98, 1417-1426.

Flamant, F., Samarut, J. (2003). Thyroid hormone receptors: lessons from knockout and knock-in mutant mice. Trends Endocrinol Metab, 14, 85-90.

Freire, C., Koifman, R.J., Sarcinelli, P.N., Simões Rosa, A.C., Clapauch, R., Koifman, S. (2013). Long-term exposure to organochlorine pesticides and thyroid status in adults in a heavily contaminated area in Brazil. Environ Res, 127, 7-15.

Friesema, E.C., Grueters, A., Biebermann, H., Krude, H., von Moers, A., Reeser, M., Barrett, T.G., Mancilla, E.E., Svensson, J., Kester, M.H., Kuiper, G.G., Balkassmi, S., Uitterlinden, A.G., Koehrle, J., Rodien, P., Halestrap, A.P., Visser, T.J. (2004). Association between mutations in a thyroid hormone transporter and severe X-linked psychomotor retardation. Lancet, 364, 1435-14377.

Goedert, M., Spillantini, M.G. (2006). A century of Alzheimer's disease. Science, 314, 777-781.

Gray, L.E. Jr, Ferrell, J.M., Ostby, J.S. (1985). Alteration of behavioral sex differentiation by exposure to estrogenic compounds during a critical neonatal period: effects of zearalenone, methoxychlor, and estradiol in hamsters. Toxicol Appl Pharmacol, 80, 127-136.

Grimaldi, L.M., Palmeri, B., Salemi, G., Giglia, G., D'Amelio, M., Grimaldi, R., Vitello, G., Ragonese, P., Savettieri, G. (2007). High prevalence and fast rising incidence of multiple sclerosis in Caltanissetta, Sicily, southern Italy. Neuroepidemiology, 28, 28-32.

Halliwell, B., Gutteridgo, J.M.C. Free radicals in biology and medicine (4th ed.). Clarendon Press, Oxford, UK, 2007.

Herbst, A.L., Green, T.H. Jr, Ulfelder, H. (1970). Primary carcinoma of the vagina. An analysis of 68 cases. Am J Obstet Gynecol, 106, 210-218.
Hill, C.E., Myers, J.P., Vandenberg, L.N. (2018). Nonmonotonic dose-response curves occur in dose ranges that are relevant to regulatory decision-making. Dose Response, 16 , 1559325818798282.

Hoffman, K., Sosa, J.A., Stapleton, H.M. (2017). Do flame retardant chemicals increase the risk for thyroid dysregulation and cancer? Curr Opin Oncol, 29, 7-13.

Holzer, G., Besson, M., Lambert, A., François, L., Barth, P., Gillet, B., Hughes, S., Piganeau, G., Leulier, F., Viriot, L., Lecchini, D., Laudet, V. (2017). Fish larval recruitment to reefs is a thyroid hormone-mediated metamorphosis sensitive to the pesticide chlorpyrifos. Elife, 6, e27595.

Hosoda, R., Nakayama, K., Kato-Negishi, M., Kawahara, M., Muramoto, K., Kuroda, Y. (2003). Thyroid hormone enhances the formation of synapses between cultured neurons of rat cerebral cortex. Cell Mol Neurobiol, 23, 895-906.

Howdeshell, K.L. (2002). A model of the development of the brain as a construct of the thyroid system. Environ Health Perspect, 110 Suppl 3, 337-348.

INERIS. (2006). Disponible sur www.ineris.fr/substances/fr/ substance/getDocument/2558.

Jacobson, J.L., Jacobson, S.W. (1996). Intellectual impairment in children exposed to polychlorinated biphenyls in utero. $N$ Engl J Med, 335, 783-789.

Jiménez-Díaz, I., Vela-Soria, F., Rodríguez-Gómez, R., ZafraGómez, A., Ballesteros, O., Navalón, A. (2015). Analytical methods for the assessment of endocrine disrupting chemical exposure during human fetal and lactation stages: a review. Anal Chim Acta, 892, 27-48.

Kadenbach, B., Barth, J., Akgun, R., Freund, R., Linder, D., Possekel, S. (1995). Regulation of mitochondrial energy generation in health and disease. Biochim Biophys Acta, 1271, 103-109.

Köhrle, J. (2000). The selenoenzyme family of deiodinase isozymes controls local thyroid hormone availability. Rev Endocr Metab Disord, 1, 49-58.

Korevaar, T.I., Muetzel, R., Medici, M., Chaker, L., Jaddoe, V. W., de Rijke, Y.B., Steegers, E.A., Visser, T.J., White, T., Tiemeier, H., Peeters, R.P. (2016). Association of maternal thyroid function during early pregnancy with offspring IQ and brain morphology in childhood: a population-based prospective cohort study. Lancet Diabetes Endocrinol, 4, 35-43.

Korevaar, T.I.M, Tiemeier, H., Peeters, R.P. (2018). Clinical associations of maternal thyroid function with foetal brain development: Epidemiological interpretation and overview of available evidence. Clin Endocrinol (Oxf), Apr 24. doi: 10.1111/cen.13724. [Epub ahead of print].

Lain, S., Trumpff, C., Grosse, S.D., Olivieri, A., Van Vliet, G. (2017). Are lower TSH cutoffs in neonatal screening for congenital hypothyroidism warranted? Eur J Endocrinol, 177, D1-D12.

Lee, E., Kim, T.H., Choi, J.S., Nabanata, P., Kim, N.Y., Ahn, M. Y., Jung, K.K., Kang, I.H., Kim, T.S., Kwack, S.J., Park, K. L., Kim, S.H., Kang, T.S., Lee, J., Lee, B.M., Kim, H.S. (2010). Evaluation of liver and thyroid toxicity in SpragueDawley rats after exposure to polybrominated diphenyl ether BDE-209. J Toxicol Sci, 35, 535-545.

Lee, J.Y., Petratos, S. (2016). Thyroid hormone signaling in oligodendrocytes: from extracellular transport to intracellular signal. Mol Neurobiol, 53, 6568-6583.

Lefebvre, J.L., Sanes, J.R., Kay, J.N. (2015). Development of dendritic form and function. Annu Rev Cell Dev Biol, 31, 741-777.

Legrand, J. (1982). Thyroid hormones and maturation of the nervous system. J Physiol (Paris), 78, 603-652. 
Li, W., Zha, J., Spear, P.A., Li, Z., Yang, L., Wang, Z. (2009). Changes of thyroid hormone levels and related gene expression in Chinese rare minnow (Gobiocypris rarus) during 3-amino-1,2,4-triazole exposure and recovery. Aquat Toxicol, 92, 50-57.

López-Juárez, A., Remaud, S., Hassani, Z., Jolivet, P., Pierre Simons, J., Sontag, T., Yoshikawa, K., Price, J., MorvanDubois, G., Demeneix, B.A. (2012). Thyroid hormone signalling acts as a neurogenic switch by repressing Sox2 in the adult neural stem cell niche. Cell Stem Cell, 10, 531-543.

Mayeux, R., Stern, Y. (2012). Epidemiology of Alzheimer disease. Cold Spring Harb Perspect Med, 2, a006239.

Mie, A., Rudén, C., Grandjean, P. (2018). Safety of safety evaluation of pesticides: Developmental neurotoxicity of chlorpyrifos and chlorpyrifos-methyl. Environ Health, 17, 77.

Miller, D.B., O'Callaghan, J.P. (2008). Do early-life insults contribute to the late-life development of Parkinson and Alzheimer diseases? Metabolism, 57 Suppl 2, S44-S49.

Millot, F., Decors, A., Mastain, O., Quintaine, T., Berny, P., Vey, D., Lasseur, R., Bro, E. (2017). Field evidence of bird poisonings by imidacloprid-treated seeds: a review of incidents reported by the French SAGIR network from 1995 to 2014. Environ Sci Pollut Res Int, 24, 5469-5485.

Min, H., Dong, J., Wang, Y., Wang, Y., Teng, W., Xi, Q., Chen, J. (2016). Maternal hypothyroxinemia-induced neurodevelopmental impairments in the progeny. Mol Neurobiol, 53, 1613-1624.

Moser, V.C., Stewart, N., Freeborn, D.L., Crooks, J., MacMillan, D.K., Hedge, J.M., Wood, C.E., McMahen, R.L., Strynar, M. J., Herr, D.W. (2015). Assessment of serum biomarkers in rats after exposure to pesticides of different chemical classes. Toxicol Appl Pharmacol, 282, 161-174.

Mughal, B.B., Fini, J.B., Demeneix, B.A. (2018). Thyroiddisrupting chemicals and brain development: an update. Endocr Connect, 7, R160-R186.

Narayan, S., Liew, Z., Bronstein, J.M., Ritz B. (2017). Occupational pesticide use and Parkinson's disease in the Parkinson Environment Gene (PEG) study. Environ Int, 107, 266-273.

Newbold, R.R., Bullock, B.C., McLachlan, J.A. (1985). Progressive proliferative changes in the oviduct of mice following developmental exposure to diethylstilbestrol. Teratog Carcinog Mutagen, 5, 473-480.

Pandey, S.P., Mohanty, B. (2015). The neonicotinoid pesticide imidacloprid and the dithiocarbamate fungicide mancozeb disrupt the pituitary-thyroid axis of a wildlife bird. Chemosphere, 122, 227-234.

Paulose, T., Speroni, L., Sonnenschein, C., Soto A.M. (2015). Estrogens in the wrong place at the wrong time: Fetal BPA exposure and mammary cancer. Reprod Toxicol, 54, 58-65.

Piaceri, I., Nacmias, B., Sorbi, S. (2013). Genetics of familial and sporadic Alzheimer's disease. Front Biosci (Elite Ed), 5, 167-177.

Pop, V.J., Kuijpens, J.L., van Baar, A.L., Verkerk, G., van Son, M.M., de Vijlder, J.J., Vulsma, T., Wiersinga, W.M., Drexhage, H.A., Vader, H.L. (1999). Low maternal free thyroxine concentrations during early pregnancy are associated with impaired psychomotor development in infancy. Clin Endocrinol (Oxf), 50, 149-155.

Rauh, V., Arunajadai, S., Horton, M., Perera, F., Hoepner, L., Barr, D.B., Whyatt, R. (2011). Seven-year neurodevelopmental scores and prenatal exposure to chlorpyrifos, a common agricultural pesticide. Environ Health Perspect, 119, 1196-1201.
Rauh, V.A., Garfinkel, R., Perera, F.P., Andrews, H.F., Hoepner, L., Barr, D.B., Whitehead, R., Tang, D., Whyatt, R.W. (2006). Impact of prenatal chlorpyrifos exposure on neurodevelopment in the first 3 years of life among inner-city children. Pediatrics, 118, e1845-e1859.

Rauh, V.A., Perera, F.P., Horton, M.K., Whyatt, R.M., Bansal, R., Hao, X., Liu, J., Barr, D.B., Slotkin, T.A., Peterson, B.S. (2012). Brain anomalies in children exposed prenatally to a common organophosphate pesticide. Proc Natl Acad Sci USA, 109, 7871-7876.

Remaud, S., Ortiz, F.C., Perret-Jeanneret, M., Aigrot, M.S., Gothié, J.D., Fekete, C., Kvárta-Papp, Z., Gereben, B., Langui, D., Lubetzki, C., Angulo, M.C., Zalc, B., Demeneix, B. (2017). Transient hypothyroidism favors oligodendrocyte generation providing functional remyelination in the adult mouse brain. Elife, 6, e29996.

Richardson, V.M., Staskal, D.F., Ross, D.G., Diliberto, J.J., DeVito, M.J., Birnbaum L.S. (2008). Possible mechanisms of thyroid hormone disruption in mice by BDE 47, a major polybrominated diphenyl ether congener. Toxicol App Pharmacol, 226, 244-250.

Rocca, W.A., Petersen, R.C., Knopman, D.S., Hebert, L.E., Evans, D.A., Hall, K.S., Gao, S., Unverzagt, F.W., Langa, K. M., Larson, E.B., White, L.R. (2011). Trends in the incidence and prevalence of Alzheimer's disease, dementia, and cognitive impairment in the United States. Alzheimers Dement, 7, 80-93.

Román, G.C., Ghassabian, A., Bongers-Schokking, J.J., Jaddoe, V.W., Hofman, A, de Rijke, Y.B., Verhulst, F.C., Tiemeier, H. (2013). Association of gestational maternal hypothyroxinemia and increased autism risk. Ann Neurol, 74, 733-742.

Schneider, G. (1973). [The determination of the larval polyphenism of the neotropical hawkmoth Erinnyis ello L. (Lepidopt., Sphingid) by various environmental factors]. Oecologia, 11, 351-370.

Silva, E., Rajapakse, N., Kortenkamp, A. (2002). Something from "nothing" - eight weak estrogenic chemicals combined at concentrations below NOECs produce significant mixture effects. Environ Sci Technol, 36, 1751-1756.

Skakkebaek, N.E., Rajpert-De Meyts, E., Main, K.M. (2001). Testicular dysgenesis syndrome: an increasingly common developmental disorder with environmental aspects. Hum Reprod, 16, 972-978.

Sohmer, H., Freeman, S. (1996). The importance of thyroid hormone for auditory development in the fetus and neonate. Audiol Neurootol, 1, 137-147.

Soldin, O.P., O'Mara, D.M., Aschner M. (2008). Thyroid hormones and methylmercury toxicity. Biol Trace Elem Res Winter, 126, 1-12.

Soto, A.M., Justicia, H., Wray, J.W., Sonnenschein, C. (1991). pNonyl-phenol: an estrogenic xenobiotic release from "modified" polystyrene. Environ Health Perspect, 92, 167-173.

Spirhanzlova, P., Le Mével, S., Du Pasquier, D., Leemans, M., Mughal, B.M., Lemkine, G., Demeneix, B., Fini, J.B. Pesticides amitrole and chlorpyrifos alter thyroid hormone and normal brain development. Soumis.

Stewart, P.W., Reihman, J., Lonky, E., Pagano, J. (2012). Issues in the interpretation of associations of PCBs and IQ. Neurotoxicol Teratol, 34, 96-107.

Syed, S. (2015). Iodine and the "near" eradication of cretinism. Pediatrics, 135, 594-596.

Tan, Z.S., Vasan, RS. (2009). Thyroid function and Alzheimer's disease. J Alzheimers Dis, 16, 503-507.

Taveggia, C., Feltri, M.L., Wrabetz, L. (2010). Signals to promote myelin formation and repair. Nat Rev Neurol, 6, 276-287. 
Tsuda, H., Hananouchi, M., Tatematsu, M., Hirose, M., Hirao, K. (1976). Tumorigenic effect of 3-amino-1H-1,2,4-triazole on rat thyroid. J Natl Cancer Inst, 57, 861-864.

US EPA. 2000a. Human health risk assessment. Chlorpyrifos. U.S. Environmental Protection Agency, Washington, DC. Available from http://www.epa.gov/oppsrrd1/op/chlor pyrifos/hedrra.

Varga, E., Nemes, C., Bock, I., Táncos, Z., Berzsenyi, S., Lévay, G., Román, V., Kobolák, J., Dinnyés, A. (2017). Establishment of an induced pluripotent stem cell (iPSC) line from a 9year old male with autism spectrum disorder (ASD). Stem Cell Res, 21, 19-22.

Vom Saal, F.S., Even, M.D., Quadagno, D.M. (1991). Effects of maternal stress on puberty, fertility and aggressive behavior of female mice from different intrauterine positions. Physiol Behav, 49, 1073-1078.

Weintraub, K. (2011). The prevalence puzzle: Autism counts. Nature, 479, 22-24.
WHO State of the science of endocrine disrupting chemicals. (2012). An assessment of the state of the science of endocrine disruptors prepared by a group of experts for the United Nations Environment Programme (UNEP) and WHO. Available from http://www.who.int/ceh/publications/endocrine/en/.

Woodruff, T.J., Zota, A.R., Schwartz, J.M. (2011). Environmental chemicals in pregnant women in the United States: NHANES 2003-2004. Environ Health Perspect, 119, 878-885.

Wu, W., Zhou, F., Wang, Y., Ning, Y., Yang, J.Y., Zhou, Y.K. (2017). Exposure to phthalates in children aged 5-7 years: Associations with thyroid function and insulin-like growth factors. Sci Total Environ, 579, 950-956.

Zheng, J., He, C.T., Chen, S.J., Yan, X., Guo, M.N., Wang, M. H., Yu, Y.J., Yang, Z.Y., Mai, B.X. (2017). Disruption of thyroid hormone (TH) levels and TH-regulated gene expression by polybrominated diphenyl ethers (PBDEs), polychlorinated biphenyls (PCBs), and hydroxylated PCBs in e-waste recycling workers. Environ Int, 102, 138-144.

Citation de l'article : Fini, J.-B. et Demeneix, B. (2019). Les perturbateurs thyroïdiens et leurs conséquences sur le développement cérébral. Biologie Aujourd'hui, 213, 17-26 